\title{
High Spatial Resolution Kelvin Probe Force Microscopy With Coaxial Probes
}

\section{Citation}

Brown, Keith A., Kevin J. Satziner, and Robert M. Westervelt. 2012. High spatial resolution Kelvin probe force microscopy with coaxial probes. Nanotechnology 23(11): 115703.

\section{Published Version}

doi:10.1088/0957-4484/23/11/115703

\section{Permanent link}

http://nrs.harvard.edu/urn-3:HUL.InstRepos:9639955

\section{Terms of Use}

This article was downloaded from Harvard University's DASH repository, and is made available under the terms and conditions applicable to Open Access Policy Articles, as set forth at http:// nrs.harvard.edu/urn-3:HUL.InstRepos:dash.current.terms-of-use\#OAP

\section{Share Your Story}

The Harvard community has made this article openly available.

Please share how this access benefits you. Submit a story.

\section{Accessibility}




\title{
High spatial resolution Kelvin probe force microscopy with coaxial probes
}

\author{
Keith A Brown, ${ }^{1, *}$ Kevin J Satzinger, ${ }^{2}$ and Robert M Westervelt ${ }^{1,3}$ \\ ${ }^{1}$ School of Engineering and Applied Sciences, Harvard University, Cambridge, MA 02138, USA \\ ${ }^{2}$ Department of Physics, Truman State University, Kirksville, MO 63501, USA \\ ${ }^{3}$ Department of Physics, Harvard University, Cambridge, MA 02138, USA \\ E-mail: westervelt@seas.harvard.edu
}

\begin{abstract}
Kelvin probe force microscopy (KPFM) is a widely used technique to measure the local contact potential difference (CPD) between an AFM probe and the sample surface via the electrostatic force. The spatial resolution of KPFM is intrinsically limited by the long range of the electrostatic interaction, which includes contributions from the macroscopic cantilever and the conical tip. Here, we present coaxial AFM probes in which the cantilever and cone are shielded by a conducting shell, confining the tip-sample electrostatic interaction to a small region near the end of the tip. We have developed a technique to measure the true CPD despite the presence of the shell electrode. We find the behavior of these probes agrees with an electrostatic model of the force, and we observe a factor of 5 improvement in spatial resolution relative to unshielded probes. Our discussion centers on KPFM, but the field confinement offered by these probes may improve any variant of electrostatic force microscopy.
\end{abstract}

PACS numbers: 07.79.Lh and 73.30.+y

\footnotetext{
${ }^{*}$ Currently at Northwestern University
} 


\section{Introduction}

Kelvin probe force microscopy (KPFM) is a widely used technique to measure the contact potential difference (CPD) between an atomic force microscope (AFM) probe and a surface [1]. KPFM has seen extensive use characterizing semiconductors [2,3], oxide material properties [4,5], photovoltaic devices [6], and biomolecules [7, 8]. KPFM is intrinsically limited by the long range of the electrostatic interaction $[9,1]$. The result of long-range contributions is that the electrostatic contribution from the region just below the tip is a small part of the entire interaction [10], and extreme care must be taken to remove topographic artifacts $[11,12]$. Despite these limitations, atomic resolution has been achieved by operating in high vacuum to make the instrument sensitive enough to detect vibration amplitude with precision $\sim 10^{-4} \mathrm{~nm}$ and frequency shifts at $\sim 5$ parts in $10^{7}[13,14,15]$. In addition, specialized probes have been developed to improve the resolution of KPFM operating in ambient conditions, such as probes with carbon nanotubes adhered to their tip [16] and cantilevers with their higher mechanical resonance modes engineered to minimally impact KPFM measurements [17].

Coaxial AFM probes can improve KPFM by confining the electric field to a small region at the tip of the AFM probe. Figure 1(a) illustrates the need for field confinement with an axisymmetric electrostatic simulation (Maxwell 2D - Ansys Inc.) in which an unshielded conducting tip is held at $1 \mathrm{~V}$ near a grounded substrate. The electrostatic potential $\phi$ varies in the whole region, denoting widespread high electric field. In contrast, figure 1(b) shows $\phi$ simulated near the tip of a coaxial probe where the core conductor is held at $1 \mathrm{~V}$ and the shell and substrate are grounded. The field is confined to the region at the tip of the probe. The improvement in confinement is evident in figure 1(c) in which the electric fields $50 \mathrm{~nm}$ below the coaxial and unshielded probes are plotted as the black and red lines respectively. Both curves are peaked directly below the probe but the field from the coaxial probe falls off much more quickly and does not exhibit the long range shoulders visible for the unshielded probe. The improvement in field confinement makes coaxial probes capable of improving the spatial resolution of any electrostatic force microscopy technique. Previously, coaxial probes have been used for scanning near field microwave microscopy [18], scanning conductance microscopy [19], and imaging with dielectrophoresis [20].

In this paper, we demonstrate enhanced spatial resolution KPFM with coaxial probes, such as the probe shown in figure 2. We begin by describing how KPFM is modified with the addition of a shell electrode and present a method to measure the true CPD despite the complication of the shell electrode. The experimental apparatus and technique used to fabricate coaxial probes are then described. Coaxial probes are calibrated to remove the effect of the shell and we find the behavior of the probes to be in agreement with our electrostatic model of coaxial probes. Finally, we demonstrate KPFM imaging with 
coaxial probes and demonstrate a factor of 5 improvement in spatial resolution relative to an unshielded probe.

\section{KPFM with coaxial probes}

KPFM provides a measurement of the contact potential difference (CPD) $v_{12}$ between a conducting AFM probe and a sample [1]. The tip and sample are capacitively coupled with capacitance $C_{12}$, and a voltage $V_{1}=V_{D C}+V_{A C} \sin (2 \pi f t)$ is applied to the probe while the sample is grounded, with potential $V_{2}=0$. The application of $V_{1}$ leads to an electrostatic force $F$ that acts to reduce the tip-sample separation $z$. The force has a component oscillating at frequency $f$ whose magnitude is given by:

$$
F_{f}=\frac{\partial C_{12}}{\partial z} V_{A C}\left(V_{D C}-v_{12}\right)
$$

By setting $f$ to the cantilever's principle vibrational resonance frequency $f_{0}$, the force $F_{f}$ drives the vibrational resonance mode of the cantilever. The vibration amplitude $A$ may be nulled by adjusting $V_{D C}$ with feedback. The value of $V_{D C}$ when $A$ is minimized is defined as the Kelvin voltage $V_{K}$, and we see that $V_{K}=v_{12}$ for a uniform sample, providing a measure of the tip-sample CPD.

The addition of a coaxial shell electrode complicates the electrostatic picture of KPFM, as shown in figure 3(a). There is now a tip-shell capacitance $C_{13}$ and a shell-sample capacitance $C_{23}$ in addition to the tip-sample capacitance $C_{12}$. The introduction of these capacitances is similar to the "stray capacitance problem" in the scanning Kelvin probe literature, a non-AFM based technique to measure CPD [21, 22, 23]. Figure 3(c) shows the capacitances in this system calculated by finite element simulation (Maxwell $2 \mathrm{D}$ - ANSYS Inc.) using the axisymmetric region shown in figure 3(b). The largest capacitance is $C_{13}$.

Care must be taken to remove the electrostatic effect of the conducting shell to perform KPFM with a coaxial probe. The electrostatic force is proportional to the derivative of capacitances with respect to tip-sample distance $z$, so we define $C_{i j}^{\prime} \equiv \frac{\partial C_{i j}}{\partial z}$. Figure 3(d) shows $C_{i j} v s . z$ calculated by the finite difference method from the capacitance data in figure 3(c). While $C_{23}$ has the largest magnitude, so long as there is no AC voltage on the shell electrode or the substrate, it will only contribute to the DC deflection. At practical separations $(z \sim 50 \mathrm{~nm}), C_{12}$ and $C_{13}$ have similar magnitudes, so the effect of $C_{13}$ must be removed to make a true measurement of the tip-sample CPD.

By adjusting the control voltages in KPFM, it is possible to perform KPFM with a coaxial probe and still attain an accurate measurement of the tip-sample CPD. We apply voltages $V_{1}=V_{A C} \sin (2 \pi f t)$ to 
the probe, $V_{2}=V_{D C}$ to the sample, and $V_{3}=V_{S}$ to the shell. Since an AC voltage is only applied to the tip, only terms with $C_{12}$ or $C_{13}$ will appear in $F_{f}$. We can again adjust $V_{D C}$ to set $A=0$ and we find the Kelvin voltage:

$$
V_{K}=-v_{12}-\frac{C_{13}}{C_{12}}\left(V_{S}+v_{13}\right),
$$

where $v_{13}$ is the tip-shell CPD. The second term is the contribution from the shell electrode, which can include topographic artifacts, because $C_{13} / C_{12}$ depends on $z$, as seen in figure 3(d). As demonstrated below, it is possible to measure the contact potential difference $v_{12}$ without the influence of the shell, by zeroing the second term by setting the shell voltage to $V_{S}=-v_{13}$.

\section{Experimental}

Coaxial AFM probes such as the one shown in figure 2 are fabricated by a technique similar to previously reported methods [24, 20, 25]. Briefly, conducting AFM probes (Arrow-NCPt — NanoWorld $\mathrm{AG}$ ) are coated with $25 \mathrm{~nm}$ of $\mathrm{Ti}$ as an adhesive layer by electron-beam evaporation (EE). An insulator is then formed by $50 \mathrm{~nm}$ of low stress silicon nitride deposited via plasma-enhanced chemical vapor deposition (PECVD). An additional $\sim 2 \mu \mathrm{m}$ thick insulating bilayer of silicon dioxide and silicon nitride is deposited with PECVD on the cantilever holder to mechanically and electrically reinforce it. Finally, a 30 $\mathrm{nm} / 50 \mathrm{~nm}$ bilayer of $\mathrm{Ti} / \mathrm{Au}$ is deposited via $\mathrm{EE}$ to form the shell electrode. The coaxial electrodes are exposed by a focused ion beam etch.

Reference samples for Kelvin probe measurements are fabricated by standard nanofabrication techniques. Chips of highly n-doped polished Si wafers (Silicon Quest International, Inc.) are diced and chemically cleaned. Large $\left(\sim \mathrm{mm}^{2}\right)$ rectangular regions are patterned with photoresist (S1813 — Shipley) using photolithography and metals are deposited with EE. Two samples are used for KPFM in this study, bare Si wafers with $20 \mathrm{~nm}$ thick $\mathrm{Cr}$ regions and Au-coated wafers with regions consisting of a $25 \mathrm{~nm} \mathrm{Pt}$ layer with a $5 \mathrm{~nm}$ Ti sticking layer.

KPFM is performed using a two-pass technique with a commercial AFM (MFP-3D - Asylum Research) with additional circuitry shown in figure 4. In the first pass, the AFM is driven mechanically and the topography is imaged by amplitude-modulation AFM. In alternating passes, the probe retraces the previously captured topographic profile and the probe is driven electrically. The feedback loop shown in figure 4 adjusts $V_{D C}$ to a value that zeroes the vibrational amplitude $A$. The value of $V_{D C}$ that minimizes $A$ is recorded as $V_{K}$. These interlaced scans are repeated to generate an image. The AFM is typically scanned at $5 \mu \mathrm{m} / \mathrm{s}$ and the KPFM retrace is at done at the same tip-sample separation $z$ as the topographic scan. All images of $V_{K}$ have the mean of each trace subtracted to reduce the effect of drift during the scan. 
For all KPFM data shown, we use the same imaging parameters which are chosen to be typical for AMKPFM done in ambient conditions. We scan a $90 \mu \mathrm{m}$ by $22.5 \mu \mathrm{m}$ region in 128 lines while recording 4096 points per line. The integral gain is set to 10 and no proportional gain. The amplitude sensitivity of each cantilever is tuned with a force-distance curve so as to set the free-space amplitude to $83 \mathrm{~nm}$ and the set-point amplitude to $50 \mathrm{~nm}$. During the second pass there is no z-offset so the tip retraces a path $50 \mathrm{~nm}$ over the substrate. During these interleaved passes, the Kelvin voltage is maintained with an integral gain of 4, no proportional gain, and an AC-voltage with amplitude of $3 \mathrm{~V}$. The typical resonance frequency of an Arrow-NCPt cantilever is found to be near $260 \mathrm{kHz}$.

In order to correctly generate the control voltages for coaxial KPFM, additional voltage sources, shown in figure 4, are added to the commercial AFM. The Asylum Research Controller (ARC) generates $V_{A C}$ and $V_{D C}$, and controls the feedback loop by measuring $A$ with optical readout of the cantilever deflection. We separate $V_{A C}$ and $V_{D C}$ with high pass and low pass filters. The AC voltage $V_{A C}$ is separated by a single pole high pass RC filter with a corner frequency at $34 \mathrm{kHz}$, which passes $99 \%$ of $V_{A C}$ to $V_{1}$. The DC voltage $V_{D C}$ is separated by a 5 pole low pass LR filter with a corner frequency at $25 \mathrm{kHz}$, which attenuates $V_{A C}$ by $\sim-80 \mathrm{~dB}$ and allows $V_{D C}$ to reach $V_{3}$. The bias voltage $V_{S}$ is generated by an external voltage source (Agilent 33220A) which is in parallel with a $10 \mu \mathrm{F}$ shunt capacitor to provide a low impedance path to ground for RF voltages. Electrical contacts to the coaxial AFM probe are provided by a custom tip holder with separate electrical contacts to the top and bottom of the probe.

\section{Results}

Before using a coaxial Kelvin probe to measure the contact potential difference (CPD), it is necessary to remove unwanted shifts in the Kelvin voltage $V_{K}$ caused by the shell. From equation (2), one can see that these shifts can be removed by adjusting the shell voltage to the value $V_{S}=-v_{13}$ where $v_{13}$ is the contact potential difference between the coaxial core and the shell. Experimentally, one can calibrate the tip voltage at this value by finding the shell voltage $V_{S}=V_{S}^{c}$ at which the measured Kelvin voltage $V_{K}$ is independent of the tip-sample separation $z$.

Figure 5 demonstrates the calibration procedure using Kelvin probe data recorded by a coaxial probe scanned above a uniform gold film. Figure 5(a) shows the mean Kelvin voltage $V_{K}$ over a $10 \times 10 \mu \mathrm{m}^{2}$ scan at a height $z=100 \mathrm{~nm}$. The Kelvin voltage $V_{K}$ increases linearly with shell voltage $V_{S}$, as predicted by equation (2); the standard deviation in $V_{K}$ is smaller than the size of the points. Figure 5(b) shows how $V_{S}$ can be calibrated by measuring $\Delta V_{K}$ vs. $V_{S}$ at three values of $z$. Here $\Delta V_{K}=V_{K}-V_{K 100}$ where $V_{K 100}=\left(5.0 \times V_{S}\right)-481 \mathrm{mV}$ is the linear fit to the data at $z=100 \mathrm{~nm}$ shown in figure 4(a). The data for $\Delta V_{K}$ vs. $V_{S}$ for three straight lines with different slopes, and they intersect at the same shell 
voltage $V_{S}^{c}=1184 \pm 1 \mathrm{mV}$. When $V_{S}=V_{S}^{c}$, the second term in equation (2) is zero, and the effect of the shell electrode is removed.

The measured slopes of $V_{K}$ vs. $V_{S}$ agree with our electrostatic model of coaxial probes: equation (2) predicts $\partial V_{K} / \partial V_{S}=C_{13}^{\prime} / C_{12}^{\prime}$. Figure 4(c) shows measurements of $C_{13} / C_{12}$ taken from the slope of the measured Kelvin voltage $V_{K} v s$. the shell voltage $V_{S}$ for five coaxial probes. To compare these measurements with theory, we use our simulations for $C_{13}$ and $C_{12}$ from figure $3(\mathrm{~d})$. We include a constant contribution to $C_{13}$ from deformation of the cantilever capacitor [26], which for identically prepared probes is $\sim 0.1 \mathrm{aF} / \mathrm{nm}$. The simulated cantilever-sample interaction is $C_{12} \sim 0.03 \mathrm{aF} / \mathrm{nm}$, roughly an order of magnitude smaller than an unshielded cantilever. The simulated ratio $C_{13} / C_{12}$ shown by the line in figure 4(c), is in good agreement with our observations.

Figure 6 compares KPFM images obtained with a coaxial probe and an unshielded conducting probe. The coaxial probe sharpens the images and eliminates artifacts by confining the electric field to a small region near the end of the probe. Figure 6(a) shows a KFPM image taken with an unshielded probe of a flat sample where the left region is gold and the right region is platinum. The measured Kelvin voltage $V_{K}$ continues to change tens of microns to the right of the step, due the long range electrostatic interaction of the cantilever with the sample. Figure 6(b) shows a KPFM image of the same sample with a coaxial probe; it exhibits a sharper transition between materials with no long range contributions. The shell electrode sufficiently shields the cantilever so its contribution is small compared to the coaxial electrodes at the tip. The improvement in the step fidelity is apparent in figure 6(c), which shows the average line trace across the transition between materials for each method.

A properly tuned coaxial KPFM measurement removes topographic artifacts common in measurements of $V_{K}$. Figure 6(d) and 6(e) show KPFM images taken with an unshielded and coaxial probe respectively of different regions of a sample with heavily doped silicon in the left region and chrome in the right region. The image taken with the coaxial probe appears smoother, which can be seen more clearly in figure 6(f) which depicts the normalized average of all 128 line traces. The red line corresponds to the unshielded probe and topographic artifacts are visible near the interface. The black line shows the data collected with the coaxial probe. It is smooth and without topographic artifacts as the calibration procedure makes this measurement technique insensitive to variation in $z$.

Coaxial probes demonstrate a factor of 5 improvement in spatial resolution compared with unshielded KPFM probes. The spatial resolution is estimated as the minimum distance $x_{\text {step }}$ required for $V_{K}$ to transition by $50 \%$ of the full value of the step from one material to another. The asymmetry of the curves makes the minimum distance necessary to change $50 \%$ of the curve a relevant measure. For 
unshielded probes, we find $x_{\text {step }}=1.6 \mu \mathrm{m}$ from figure $6(\mathrm{c})$ and $x_{\text {step }}=1.7 \mu \mathrm{m}$ from figure $6(\mathrm{f})$. Coaxial probes offer a large improvement, giving $x_{\text {step }}=410 \mathrm{~nm}$ from figure $6(\mathrm{c})$ and $x_{\text {step }}=280 \mathrm{~nm}$ from figure 6(f), commensurate with the diameters of the coaxial probes used in each measurement.

\section{Discussion}

Simulations of the electric field profile produced by unshielded and coaxial probes above a conducting substrate are shown in figures 7(a) and 7(b) for a range of tip-sample separations z. These simulations show that the spatial resolution of coaxial probes is determined by the tip geometry, and demonstrate how a coaxial probe can be resistant to topographical artifacts. For the unshielded probe (figure 7(a)), the electric field distribution narrows considerably as $\mathrm{z}$ is decreased from $50 \mathrm{~nm}$ to $10 \mathrm{~nm}$. Decreasing $\mathrm{z}$ improves the spatial resolution, but this also means that variations in $\mathrm{z}$ will strongly affect the point-spread function of the measurement, leading to topographical artifacts. In addition, broad shoulders to the electric field profile still exist when the height $\mathrm{z}$ is equal to the tip radius of $10 \mathrm{~nm}$, adding long-range contributions to any electrostatic measurement. For the coaxial probe (Fig. 7(b)), decreasing $\mathrm{z}$ causes the electric field profile to adopt a nearly trapezoidal shape with a width that is simply the diameter of the core conductor. Away from the core, the field rapidly decays toward zero across the insulator thickness. The relative insensitivity of the field profile to $\mathrm{z}$ makes this technique resistant to topographical artifacts.

We have demonstrated that coaxial AFM probes can perform Kelvin probe force microscopy with enhanced spatial resolution. A theoretical model for performing KPFM with a coaxial tip to measure the tip-sample contact potential difference has been developed. Coaxial AFM probes with $50 \mathrm{~nm}$ electrode spacing at the tip were fabricated and tested with a commercial AFM. Unwanted shifts in the Kelvin voltage by the shell electrode can be avoided by calibrating the shell bias voltage $V_{S}$ to give the same Kelvin voltage $V_{K}$ at different tip-sample separations $z$. We find that coaxial probes have the following advantages: they reduce capacitive coupling between the cantilever and the sample, decrease topographical artifacts, and improve the spatial width of a transition from one material to another, from $\sim 1.6 \mu \mathrm{m}$ to $\sim 300 \mathrm{~nm}$ in our experiments. The spatial resolution of a coaxial tip can be improved by creating tips with thinner insulating layers and finer centre conductors. The dielectric breakdown strength of SiNx films is $\sim 0.5 \mathrm{~V} / \mathrm{nm}$,[27] so insulators as thin as $10 \mathrm{~nm}$ would be sufficient to withstand typical voltages in an KPFM measurement. One method for creating centre conductors of nearly atomic sharpness would be to polish the multilayer tip on an abrasive conducting surface during fabrication until electrical contact is made between the centre conductor and the surface. The blunt shape of these tips could be a disadvantage for topographical imaging, although for the relatively flat samples studied here, 
we did not notice any degradation in topographic image quality. In order to preserve tip sharpness for topographical imaging, one could mill the tip at an angle or use a chemical etch to create retain a protruding centre conductor.

Coaxial probes improve the spatial resolution by confining the electric field to a region near the end of the tip which means that coaxial probes promise to improve the quality of any imaging technique that is based on the electrostatic interaction. It is important to reiterate that this is a demonstration of the improved performance of a coaxial probe over a standard probe rather than an attempt to surpass state of the art resolution in KPFM measurements. The mm-scale resolution resulting from the imaging parameters and unshielded probe used in this work are not uncommon for ambient AM-KPFM measurements.[28] Lower drive amplitudes or decreased tip-sample separations are also commonly used and provide greatly improved resolution.[29] As discussed in the introduction, many techniques exist for performing KPFM with enhanced resolution[13, 14, 15] including reducing the tip-sample separation,[28] operating in vacuum,[30] and using frequency modulation (FM) rather than amplitude modulation.[28,30] Coaxial probes may be used in vacuum and are compatible with FM-KPFM so in principle they could be used to improve the spatial resolution in these modalities. As a further potential improvement, it is possible to employ a one-pass technique where the surface potential and topography are measured simultaneously.[14]

\section{Acknowledgements}

We acknowledge support by the Department of Defense through a National Defense Science \& Engineering Graduate (NDSEG) Fellowship, the National Nanotechnology Infrastructure Network through the Research Experience for Undergraduates Program through National Science Foundation grant number ECCS-0821565, and the Department of Energy through grant number DE-FG02-07ER46422.

\section{References}

[1] Jacobs H O, Knapp H F and Stemmer A 1999 Rev. Sci. Instrum. 701756

[2] Oliver R A 2008 Rep. Prog. Phys. 71076501

[3] Benstetter G, Biberger R and Liu D 2009 Thin Solid Films 5175100

[4] Kalinin S V, Shao R and Bonnell D A 2005 J. Am. Ceram. Soc. 881077

[5] Jaramillo R and Ramanathan S 2011 Sol. Energy Mater. Sol. Cells 95602

[6] Hoppe H, Glatzel T, Niggemann M, Hinsch A, Lux-Steiner M C and Sariciftci N S 2005 Nano Lett. 5 269

[7] Sinensky A K and Belcher A M 2007 Nat. Nanotechnol. 2653 
[8] Leung C, Maradan D, Kramer A, Howorka S, Mesquida P and Hoogenboom B W 2010 Appl. Phys. Lett. 97203703

[9] Belaidi S, Lebon F, Girard P, Leveque G and Pagano S 1998 Appl. Phys. A 66 S239

[10] Jacobs H O, Leuchtmann P, Horman O J and Stemmer A 1998 J. Appl. Phys. 841168

[11] Okamoto K, Sugawara Y and Morita S 2002 Appl. Surf. Sci. 188381

[12] Machleidt T, Sparrer E, Kapusi D and Franke K-H 2009 Meas. Sci. Technol. 20084017

[13] Sommerhalter C, Matthes T W, Glatzel T, Jager-Waldau A and Lux-Steiner M C 1999 Appl. Phys. Lett. 75286

[14] Silveira W R, Muller E M, Ng T N, Dunlap D H and Marohn J A 2006 High-sensitivity electric force microscopy of organic electronic materials and devices (Scanning Probe Microscopy: Electrical and Electromechanical Phenomena at the Nanoscale vol III) ed S V Kalinin and A Gruverman. (New York: Springer Verlag) pp 788830

[15] Sadewasser S, Jelinek P, Fang C-K, Custance O, Yamada Y, Sugimoto Y, Abe M and Morita S 2009 Phys. Rev. Lett. 103266103

[16] Zhao M, Sharma V, Wei H, Birge R R, Stuart J A, Papdimitrakopoulos F and Huey B D 2008 Nanotechnology 19235704

[17] Sadewasser S, Villanueva G and Plaza J A 2006 Appl. Phys. Lett. 89033106

[18] Rosner B T and Weide D W v d 2002 Rev. Sci. Instrum. 732505

[19] Noh J H, Nikiforov M, Kalinin S V, Vertegel A A and Rack P D 2010 Nanotechnology 21365302

[20] Brown K A, Berezovsky J and Westervelt R M 2011 Appl. Phys. Lett. 98183103

[21] Surplice N A and D'Arcy R J 1970 J. Phys. E: Sci. Instrum. 3477

[22] Baikie I D, Venderbosch E, Meyer J A and Estrup P J 1991 Rev. Sci. Instrum. 621142075

[23] Nabhan W, Broniatowski A, De Rosny G and Equer B 1994 Microsc. Microanal. Microstruct. 5509

[24] Brown K A, Aguilar J A and Westervelt R M 2010 Appl. Phys. Lett. 96123109

[25] Brown K A and Westervelt R M 2011 Nano Lett. 113197

[26] Brown K A, Yang B and Westervelt R M 2011 Appl. Phys. Lett. 100053110

[27] Stryahilev D and Nathan A 2002 J. Vac. Sci. Technol. A 201097

[28] Ziegler D and Stemmer A 2011 Nanotechnology 22075501

[29] Liscio A, Vincenzo P and Samorì 2008 Adv. Funct. Mater. 18907

[30] Zerweck U, Loppacher C, Otto T, Grafstrom S and Eng L M 2005 Phys. Rev. B: Condens. Matter 71 125424 


\section{Figure Captions}
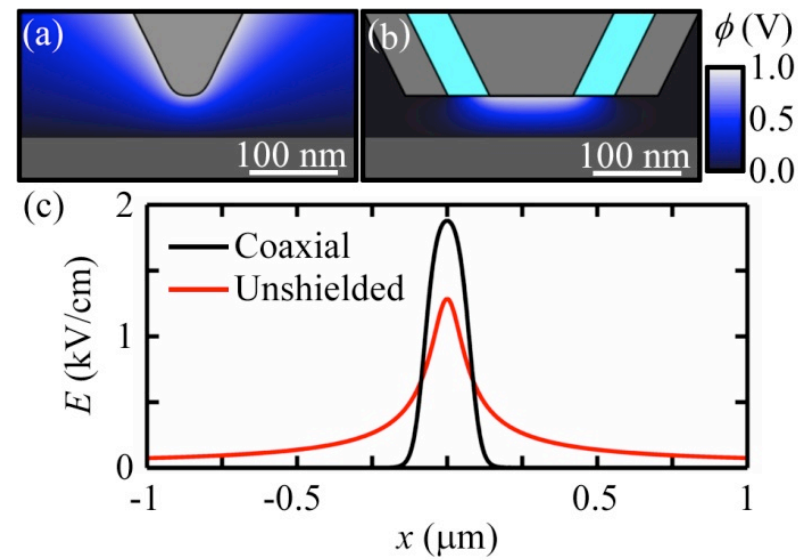

Figure 1. (a) Simulation of the electrostatic potential $\phi$ near the tip of an unshielded conducting probe. The probe is held at $1 \mathrm{~V}$, while the substrate is grounded. The potential $\phi$ is seen to vary over the entire region. (b) Electrostatic simulation of the potential $\phi$ for a coaxial probe, showing that $\phi$ only varies immediately below the tip of the core conductor. The core conductor is held at $1 \mathrm{~V}$ while the substrate and shell electrode are grounded. (c) Electric field amplitude $E$ at a distance $50 \mathrm{~nm}$ below the tip of a coaxial and an unshielded probe $v s$. distance $x$ away from the axis of the probe. The field created by the coaxial probe is both stronger and more localized, and does not have the broad shoulders of the unshielded probe. 


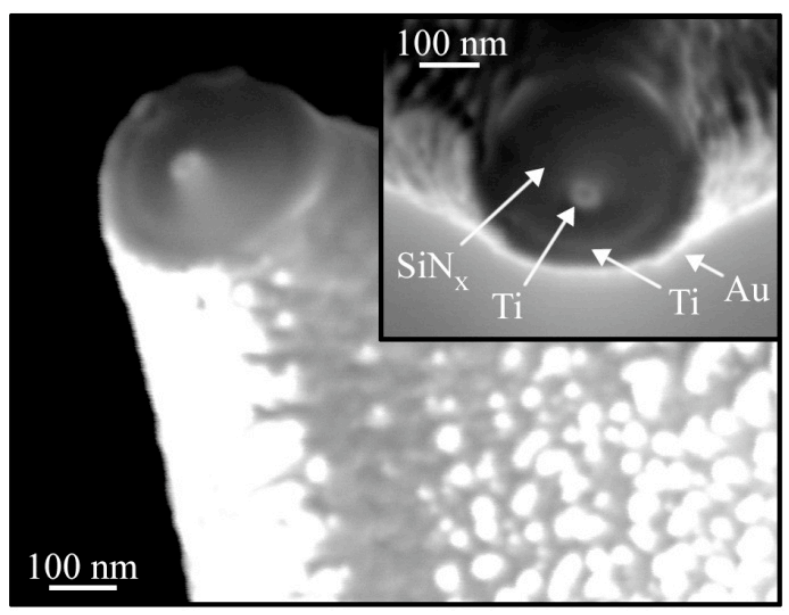

Figure 2. Scanning electron micrograph of a coaxial probe. (inset) The thin films that form the shell $(\mathrm{Ti} / \mathrm{Au})$, the insulating layer $\left(\mathrm{SiN}_{\mathrm{x}}\right)$, and the core electrode (Ti) are visible at the tip.
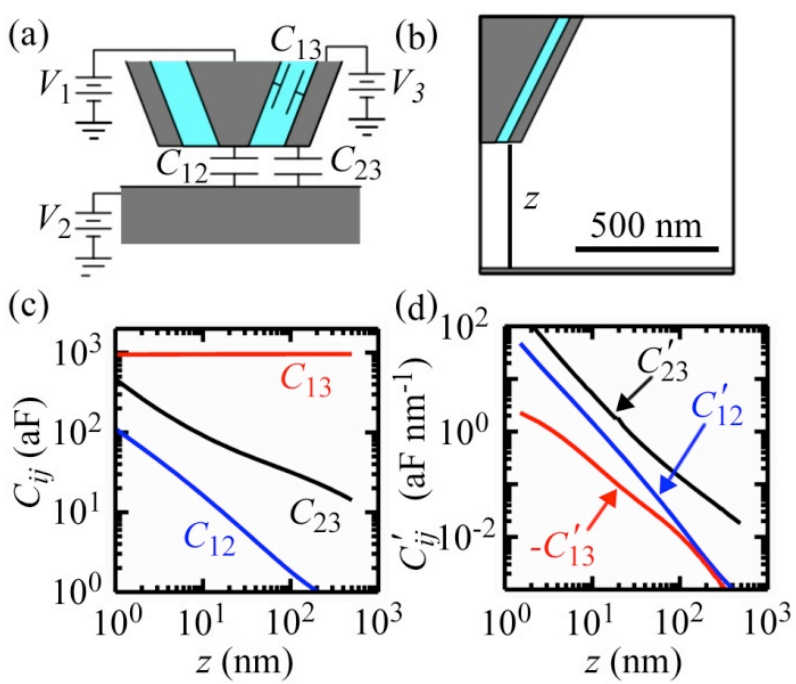

Figure 3. (a) Diagram showing the capacitances $C_{i j}$ and applied voltages $V_{i}$ of the coaxial tip-sample system. (b) Scale schematic of the region used to calculate $C_{i j}$ using axisymmetric finite-element simulations (Maxwell 2D - Ansys Inc.). (c) Simulated capacitances $C_{i j}$ of a coaxial tip vs. distance $z$ away from a substrate. The tip-shell capacitance $C_{13}$ is the largest. (d) The magnitude of the capacitance derivatives $C_{i j}^{\prime}$ vs. $z$ calculated from the simulations in (c). The derivatives $C_{12}$ and $C_{23}$ are negative, while the derivative $C_{13}$ is positive. 


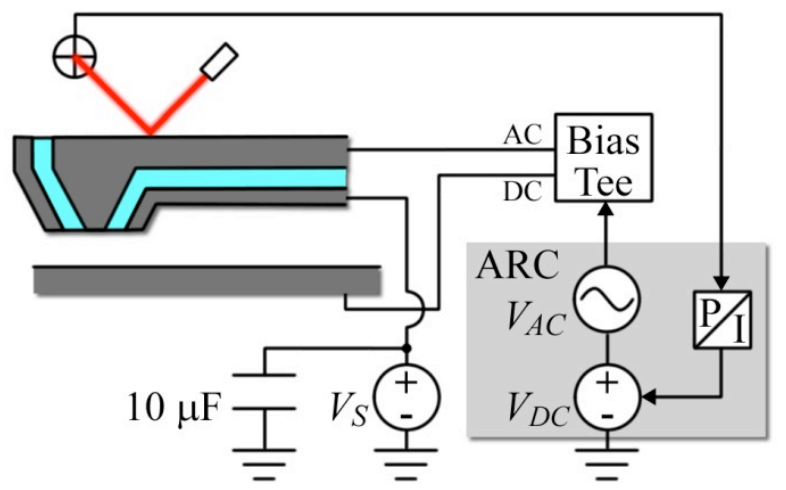

Figure 4. Schematic of the coaxial KPFM apparatus. The Asylum Research Controller (ARC) creates the AC excitation voltages $V_{A C}$ and measures the cantilever vibrational amplitude $A$ which controls the DC voltage $V_{D C}$ forming a feedback loop. The voltages $V_{A C}$ and $V_{D C}$ are separated with a bias tee to route $V_{D C}$ to the sample while $V_{A C}$ is directed to the core conductor. The shell bias voltage $V_{S}$ is created by an external voltage supply and tied to ground with a $10 \mu \mathrm{F}$ capacitor to ensure $V_{A C}$ is not present on the shell electrode. 

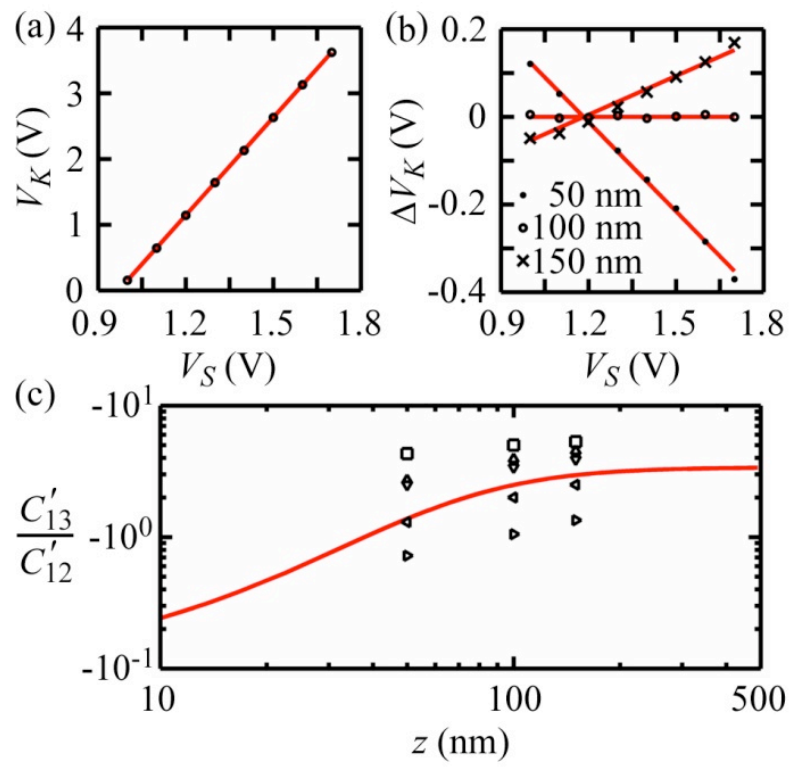

Figure 5. Procedure to calibrate a coaxial tip for Kelvin probe force microscopy to remove the effects of the shell, showing KPFM measurements of a uniform gold film by a coaxial tip at tip-sample distance $z=$ $100 \mathrm{~nm}$. (a) Measured Kelvin voltage $V_{K} v s$. shell bias voltage $V_{S}$; the measured points show the linear dependence on $V_{S}$ predicted by equation (2). (b) Kelvin voltage $\Delta V_{K} v s . V_{S}$ at three different tip-sample separations $z$; here the difference $\Delta V_{K}=V_{K}-V_{K 100}$ between $V_{K}$ and the linear fit $V_{K 100}$ for $z=100 \mathrm{~nm}$ is shown. The coaxial probe is calibrated at the shell voltage $V_{S}=V_{S}^{c}$ where the three lines intersect. (c) Experimental ratios $C_{13} / C_{12}$ vs. $z$ for the derivatives of the core-shell and core-sample capacitances $C_{13}$ and $C_{12}$ for five different coaxial probes, compared with the theoretical expression for coaxial probes. 

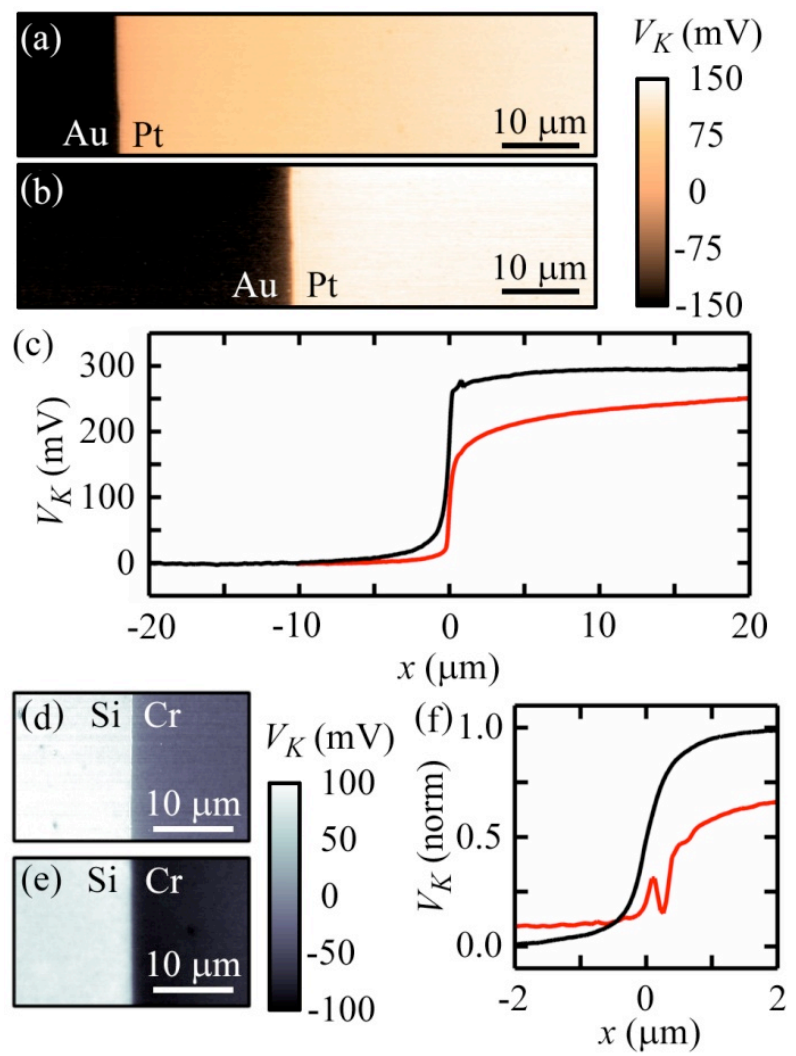

Figure 6. (a) KPFM image taken with an unshielded probe of sample with a gold region on the left and a platinum region on the right. The Kelvin voltage $V_{K}$ is seen to vary over tens of microns asymmetrically due to the contribution of the cantilever. (b) KPFM image of the same sample with a coaxial probe. The Kelvin voltage $V_{K}$ quickly transitions to the saturated value and the step appears symmetric. (c) Average line traces from (a) and (b) showing the step function of an unshielded probe (red line) and a coaxial probe (black line). The unshielded probe transitions very slowly after the step due to the contribution from the cantilever whereas the coaxial probe quickly saturates. (d) KPFM image taken with an unshielded probe of a sample with a heavily doped silicon region on the left and a chrome region on the right. (e) KPFM image of the same sample taken with a coaxial probe. (f) Average line traces from (d) and (e) showing the step function of an unshielded (red line) and a coaxial probe (black line). The unshielded probe exhibits topographical artifacts at the interface of the two materials whereas the coaxial probe exhibits a smooth transition. 

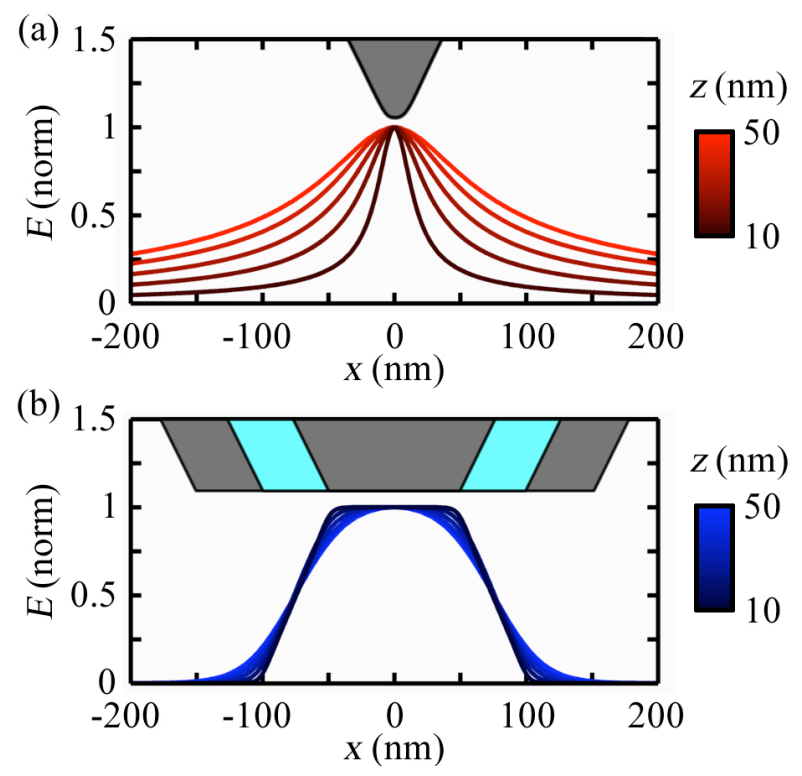

Figure 7. Axisymmetric electrostatic simulations of the electric field on a conducting substrate when an unshielded (figure 7(a)) or coaxial (figure 7(b)) probe is held at a height $\mathrm{z}$ above the substrate. Each panel shows a scaled schematic diagram of the probe tip used for the simulation. The unshielded probe and core conductor of the coaxial probe are held at $1 \mathrm{~V}$ while the substrate and shell of the coaxial probe are grounded. The electric field of each curve is normalized by its maximum value for ease of comparison. 